\title{
Studies of the microwave emission of extensive air showers with GIGAS and MIDAS at the Pierre Auger Observatory
}

\author{
Romain Gaïor $^{* a}$ for the Pierre Auger Collaboration ${ }^{b}$, and Matthew Richardson ${ }^{c}$ \\ ${ }^{a}$ Laboratoire de Physique Nucléaire et de Hautes Energies (LPNHE), Universités Paris 6 et Paris \\ 7, CNRS-IN2P3, Paris, France \\ ${ }^{b}$ Observatorio Pierre Auger, Av. San Martín Norte 304, 5613 Malargüe, Argentina \\ ${ }^{c}$ Vanderbilt University, USA \\ E-mail: auger_spokespersons@fnal.gov \\ Full author list: http://www.auger.org/archive/authors_icrc_2017.html
}

In 2008, a radio signal interpreted as Molecular Bremsstrahlung Radiation (MBR) was detected at SLAC at microwave frequencies from electromagnetic showers produced in beam test experiments. Due to the isotropic nature of MBR and its insensitivity to atmospheric attenuation and light conditions, it would allow the measurement of the shower longitudinal profile with an almost $100 \%$ duty cycle compared to $15 \%$ at most with the fluorescence technique today. Several experiments either in the laboratory or in situ within cosmic-ray observatories have been set up aiming at the detection of the MBR flux. The Pierre Auger Observatory has been used as the base for two experiments pursuing the detection of the MBR at $\mathrm{GHz}$ frequencies. MIDAS is a radio telescope instrumented with a parabolic dish focusing the radio signal on an array of 53 horn antennas and has taken data for 2 years. GIGAS on the other hand is a single antenna detector embedded in a surface detector. It was implemented in three different versions with a gradually improved sensitivity to comply with the evolution in the expected MBR intensity. We review these two experimental efforts undertaken at the Pierre Auger Observatory attempting at MBR detection and present their latest results.

35th International Cosmic Ray Conference - ICRC2017

10-20 July, 2017

Bexco, Busan, Korea

${ }^{*}$ Speaker. 

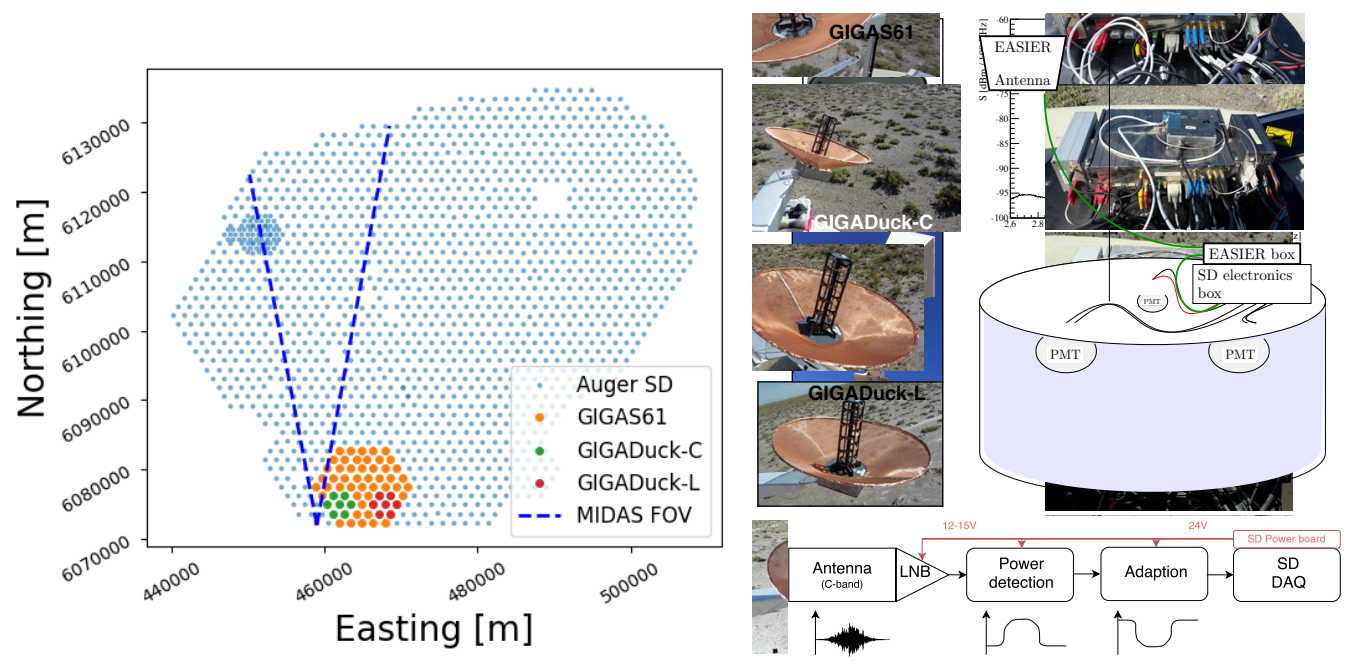

Figure 1: Left: The GIGAS arrays and MIDAS field of view overlaid with the surface detector of the Pierre Auger Observatory. Right: Scheme of GIGAS concept. The sensor placed on the tank is one the three antenna shown in the left side. The signal chain common for all the setups is represented on the bottom part.

\section{Introduction}

The measurement of the composition of Ultra High Energy Cosmic Rays (UHECR) is crucial to understand their origin. The Pierre Auger Observatory operates currently two main detectors to measure the properties of the Extensive Air Showers (EAS) induced by the UHECR. The Surface Detector (SD) is an array of over 1660 water-Cherenkov detectors (WCD), it measures the particle density at the ground. The Fluorescence Detector (FD) measures the fluorescence light emitted by EAS from 5 sites surrounding the SD. While the SD is limited in its sensitivity to the mass composition, the FD is sensitive to it via the measurement of the longitudinal profile but can be operated only with a duty cycle of $15 \%$. The development of an additional particle detector complementing the existing one and improving its mass sensitivity is the focus of the current upgrade effort undertaken at the Pierre Auger Observatory [1].

New detection channels have also been considered. The observation of the EAS through the radio waves emitted along its development is one of them. In the VHF band (around $50 \mathrm{MHz}$ ) the radio signal from EAS is dominated by the coherent emission beamed around the shower axis within the Cherenkov cone [2], preventing the measurement of the longitudinal development.

A promising technique was proposed in 2008 [3] after the observation in a beam test of a signal in the microwave frequencies upon the passage of a particle shower in an anechoic chamber. This signal interpreted as Molecular Bremsstrahlung Radiation (MBR), is emitted isotropically and would allow one to measure the longitudinal profile of the EAS, like with the fluorescence technique but with a $100 \%$ duty cycle. Despite the efforts to measure this radiation in subsequent beam tests, or in in situ experiment, the intensity of the MBR was not confirmed $[4,5,6]$. In this contribution we present the development and the results of two radio experiments, GIGAS and MIDAS, installed within the Pierre Auger Observatory (see Figure 1 left). For each experiment we will describe the detector characteristics and then present a search for radio event in coincidence with the Auger SD. 


\section{GIGAS}

\subsection{Detectors}

Concept GIGAS (GHz Identification of Giant Air Shower) is designed to observe the radio emission from EAS with an antenna looking up in the sky. Each radio detector is embedded in a WCD and thus takes advantage of the trigger but also of the solar power system and the data acquisition. The detector is composed of an antenna followed by an amplification and a filtering stage. The radio frequency (RF) signal is then transformed into its power envelope with a logarithmic amplifier which is in turn scaled to fit into the Auger SD front end where the GIGAS signal replaces a low gain channel of one of the three PMTs. The scheme of the GIGAS detector is shown in Figure 1 (right). This concept has been implemented in three different versions, two in the C-band (3.4 to $4.2 \mathrm{GHz}$ ) GIGAS61 and GIGADuck-C and one the L band (1 to $1.4 \mathrm{GHz}$ ), GIGADuck-L (see Figure 1 (right)).

GIGAS61 The GIGAS61 is the first array installed in the Pampa. A test bed of 7 antennas was installed in April 2011 and the completion to 61 detectors took place a year later. Each detector is composed of a C-band cylindrical horn antenna with a half power beam width (HPBW) of $90^{\circ}$. The antenna points toward the zenith. GIGAS61 has measured clear events in coincidence with the particle detector (see section 2.2). However, because of the short distance to the shower axis the origin of this signal can be attributed to coherent processes and cannot be evidence for the MBR.

GIGADuck-C GIGADuck is an array of seven detectors instrumented with a larger gain antenna to increase the sensitivity, and an optimized geometry to enhance the coincidence probability between radio detectors. Such a coincidence would favor the MBR origin of a signal against a coherent process. In this modified geometry, each antenna points in a different direction, the central one is pointed at the zenith while the other six are tilted by $20^{\circ}$ and have their azimuth oriented in the direction of the central detector. In the C-band, the antenna is a pyramidal horn with $15 \mathrm{~dB}$ gain and a HPBW of $60^{\circ}$, followed by an LNB (Norsat 8115F).

GIGADuck-L The GIGADuck design has been also implemented in the L-band. The antenna is a helix antenna with a maximum gain at $1.4 \mathrm{GHz}$ and a HPBW of $60^{\circ}$. Contrary to the two other setups, the amplification board was developed in laboratory. It integrates a band pass filter and an electric surge protection and two commercial LNA chips (Broadcom Limited MGA633P8 and MGA13116) in series. The gain and noise temperature of this board were characterized before the installation. The gain in the bandwidth is around $50 \mathrm{~dB}$ and the noise temperature ranges from 60 to $80 \mathrm{~K}$ among the 9 boards tested.

Detector calibration The sensitivity of a radio power detector like GIGAS can be estimated with the figure of merit that defines approximately the minimum flux one can detect, $F_{\min }=$ $k_{\mathrm{B}} T_{\text {sys }} / A_{\text {eff }} \sqrt{\Delta v \Delta t}$ where $k_{\mathrm{B}}$ is the Boltzmann constant, $T_{\text {sys }}$ the system temperature, $A_{\text {eff }}$ the effective area, $\Delta v$ and $\Delta t$ the bandwidth and the time over which the signal can be integrated.

The comparison of the simulated $A_{\text {eff }}$ of the three GIGAS detectors in the figure 2 (left) shows the direct increase in sensitivity from this parameter.

The system temperature is more difficult to estimate. For the three setups it was indeed measured 

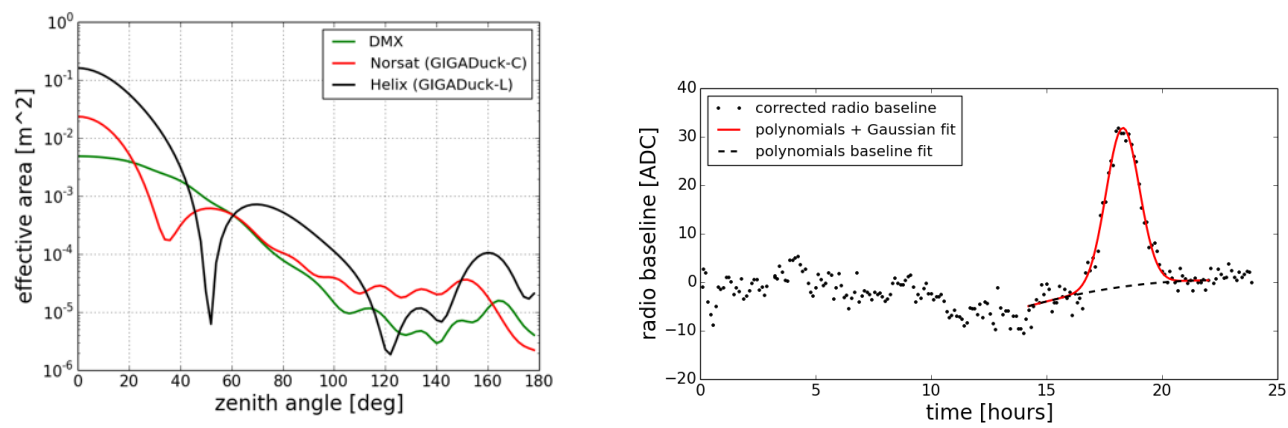

Figure 2: Left: Antenna effective area as a function of the zenith angle for the three setups. Right: Example of a daily baseline corrected from the outside temperature dependence.

with different methods. For GIGAS61 detectors it was estimated by applying the so called Y-factor method on a dedicated measurement on site. We simply measured the power output when the antenna was pointed towards the sky and then when it was oriented towards the ground, i.e. two different sources of microwaves emission. From the difference of power between these measurements, an intrinsic noise system temperature of $120 \mathrm{~K}$ was measured.

For the GIGADuck-C band, we have developed a method using the Sun as a calibration source. The emission from the Sun produces a bump in the radio baseline recorded in the monitoring system. After correcting the baseline from outside temperature dependence, we can fit this bump (see an example in Figure 2). We apply this method on a set of daily baselines selected on the quality of the radio data and the quality of the fit. The system temperature is deduced from the comparison with the expected amplitude of the Sun contribution estimated with the solar flux based on observations at the Nobeyama Radio Observatory $(\mathrm{NRO})^{1}$ at $3.75 \mathrm{GHz}$. By comparing the measured and simulated values of the amplitude of the bump but also the time of its maximum, the system noise temperature and the pointing direction of the antenna were measured simultaneously. We found system noise temperatures ranging from 54 to $61 \mathrm{~K}$ and a angular distance from the nominal pointing of a few degrees with a maximum of $12^{\circ}$.

The baseline of the GIGADuck-L detectors exhibits also a clear bump attributed to the Sun. However other contributions are noticed over the day and prevent us from isolating the Sun signal (a possible origin of these contributions are the positioning satellite like GPS which all emit in the Lband). Therefore the system temperature is directly measured from the baseline level in monitoring. This procedure is made possible thanks to the calibration carried out prior to the installation. Noise temperatures ranging from 95 to $145 \mathrm{~K}$ were found for the seven installed detector. This accounts also for the noise induced by the Sun and other sources, explaining the spread in the temperature.

\subsection{Event search}

We show in this section a search for radio events in coincidence with EAS recorded by the Auger SD. The radio data set is composed of the three setups and their operation time overlap from April 2011 to May 2017. We select high quality SD events, by applying regular selection criteria

\footnotetext{
${ }^{1}$ The Nobeyama Radio Polarimeters is operated by Nobeyama Radio Observatory, a branch of National Astronomical Observatory of Japan.
} 

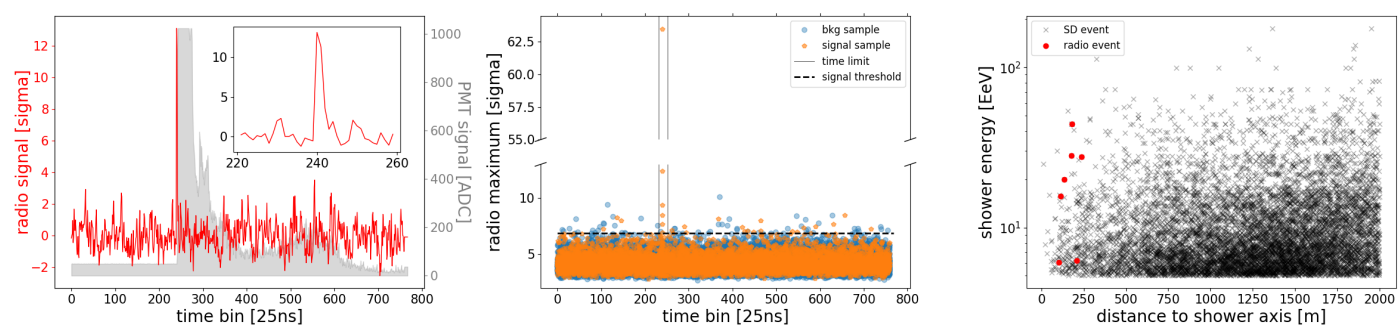

Figure 3: Left: radio waveform (normalized by the trace standard deviation). Middle: The radio maxima of the background and signal data sets. Right: event distribution in the energy / distance plane. The red dots are the event identified in radio.

Table 1: Characteristics of the radio events detected with GIGAS.

\begin{tabular}{rccccccc}
\hline Event date & $2011 / 06 / 30$ & $2012 / 09 / 05$ & $2013 / 01 / 03$ & $2013 / 06 / 04$ & $2013 / 07 / 16$ & $2013 / 09 / 26$ & $2015 / 02 / 03$ \\
\hline Energy [EeV] & 16 & 6 & 27 & 19.5 & 42 & 6.2 & 28 \\
Distance [m] & 112 & 103 & 237 & 133 & 181 & 208 & 176 \\
$(\theta, \phi)$ & $(29.6,-17)$ & $(48,167)$ & $(55,34)$ & $(53,-1.2)$ & $(40,155)$ & $(59,-49)$ & $(54,1)$ \\
Radio maximum & 13 & 7.7 & 12.3 & 6.9 & 63 & 8.5 & 9.4 \\
\hline
\end{tabular}

on the shower reconstruction and by removing the events tagged as lightning. A second set of cuts is then applied on the radio traces: we remove traces with large RMS or with more than 10 bins saturated (out of 768). The data set is split in a background set composed of the WCD with a distance from the shower axis larger than $3 \mathrm{~km}$ and a signal set which selects, among the EAS event of energy larger than $5 \mathrm{EeV}$ and zenith angle smaller than $60^{\circ}$, the WCD closer than $2 \mathrm{~km}$ from the shower axis. The former is not expected to contain any detectable radio event while the latter should be enriched in them. The radio waveform is linearized converted in unit of it standard deviation (see for instance Figure 3 (left)), the distribution of the radio maximum as a function of the time of this maximum is shown in Figure 3 (middle) for the GIGAS61 setup. One can see a clear accumulation of events with large signal to noise ratio at the time bin 240 which is only $50 \mathrm{~ns}$ before the SD trigger time (see Figure 3 (left)). To extract the radio events, we determine the value of the radio maximum that holds $99.7 \%$ of the events in the background set and we set this value as threshold for the signal set. The time of maximum is also required to be inside a $500 \mathrm{~ns}$ window around the particle trigger to absorb any possible time delay induced by the detector. Seven radio events were found, all detected with the GIGAS61 setup. A typical waveform is represented in Figure 3 (left), the radio signal (in red) is a high and short pulse of less than $50 \mathrm{~ns}$ long (see inset of Figure 3 (left)) coincident with the PMT signal start (in grey). The parameters of these events are listed in Table 1. A striking feature is the short distance to the shower axis of all the detected events. Indeed, among the high energy events recorded by the Auger SD, no signal at larger distances than $250 \mathrm{~m}$ induced a clear signal in GIGAS setups (Figure 3 (right)). 


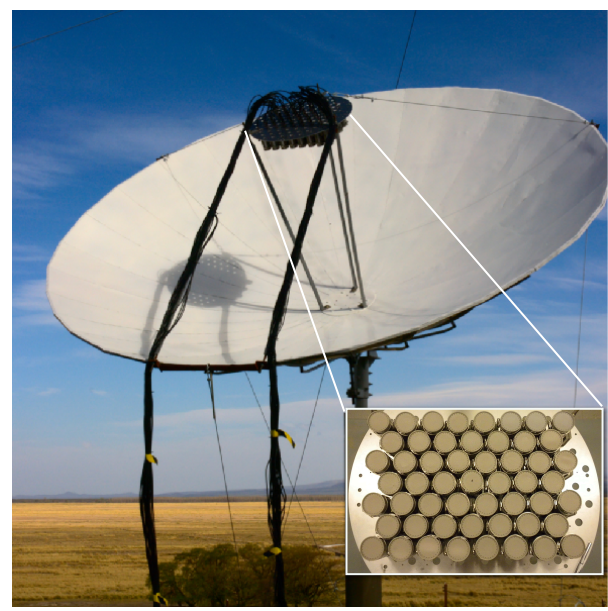

Figure 4: MIDAS detector installed in the Pampa at the Pierre Auger Observatory.

\section{MIDAS}

\subsection{Detectors}

The MIDAS detector, first commissioned at the University of Chicago in 2010 [7], was installed at the Pierre Auger Observatory in 2012, with its field of view covering a portion of the SD (Figure 1.) The telescope consists of a $5 \mathrm{~m}$ diameter parabolic antenna with a 53 pixel camera at its focus (Figure 4) covering a field of view of approximately $20^{\circ} \times 10^{\circ}$. Each pixel is composed of a feed horn, a low noise amplifier, and a frequency down converter. An RF power detector converts radio waves detected by a pixel to a voltage level which is digitized by a 14 bit analogto-digital converter (ADC) at a sampling rate of $20 \mathrm{MHz}$ (an ADC board serves 16 pixels.) Up to 2048 samples are stored in a circular buffer and processed by a first level trigger (FLT) algorithm implemented in the on-board field programmable gate array (FPGA). A second level trigger (SLT) decision is taken by a Master Trigger board by searching for 4-pixel patterns compatible with a track-like signal from an EAS. When an SLT is present, all 53 pixels are readout [7].

\subsection{Event search and limits on MBR intensity}

Event search A search for MBR signal from EAS based on time coincidence between MIDAS and SD events was performed with data collected from September 14, 2012 to September 26, 2014. Notice that the MIDAS telescope is self-triggered, contrary to GIGAS where the trigger is provided by the SD local station. Thus a first selection was performed to reject periods with high level of radio noise where the SLT rate can reach several $\mathrm{kHz}$, to be compared with an expected SLT rate from white noise accidentals of less than a mHz. To remove these periods, the SLT rate was required to be less than $0.5 \mathrm{~Hz}$. Also, periods when the FLT rate exceeded $2.4 \mathrm{kHz}$ were removed. These criteria resulted in an active MIDAS observational time $t_{\mathrm{obs}}$ of about 359 days.

High quality SD events were selected by requiring the tank with the largest signal to be surrounded by active tanks and a reconstructed shower energy above $1 \mathrm{EeV}$. Also, the SD reconstructed shower core was required to be within the MIDAS field of view projected on the ground. Lastly, a search was performed for MIDAS and SD events occurring in a coincidence time window of $\pm 300 \mu \mathrm{s}$, a 
relatively large window to account for time delays due to microwave signal propagation. Only one event was found to fulfill all the selection criteria.

The expected rate $r_{\mathrm{c}}$ from random coincidences can be estimated by $r_{\mathrm{c}} \simeq r_{\mathrm{A}} r_{\mathrm{M}} \tau$ where $r_{\mathrm{A}}=$ $8.9 \times 10^{-4} \mathrm{~Hz}$ and $r_{\mathrm{M}}=1.8 \times 10^{-2} \mathrm{~Hz}$ are the measured Auger and MIDAS event rate medians, and $\tau=600 \mu \mathrm{s}$ is the coincidence time interval. The expected number of coincidence events is then given by $N_{\mathrm{c}}=r_{\mathrm{c}} t_{\mathrm{obs}} \approx 0.3$. A similar estimate was obtained by repeating the analysis on mock samples where the SD event detection time was randomly shifted (see [8].) Thus, the single found candidate event is consistent with expectations from purely random coincidences. The waveforms of the SLT pixels associated with the candidate event are shown the Figure 5 (left), with the full pixel camera signals in the inset. The signal time characteristics and the multiple pixels patterns observed in the MIDAS camera are not compatible with an EAS event. In addition, the SD reconstructed shower has an energy of $2.5 \mathrm{EeV}$ and a core located at $53 \mathrm{~km}$ distance from the MIDAS telescope. Since many more SD events of larger energy and smaller distance are present in the selected sample but not detected in coincidence with MIDAS, it is highly unlikely that the candidate event is real. Thus the candidate event was rejected and the search ended with a null result.

Limits on MBR properties Dedicated simulations were performed to establish limits on the properties of MBR from EAS. Following [3], we parameterize in the simulation the microwave flux of the EAS at the MIDAS detector, $I_{\mathrm{f}}$, as

$$
I_{\mathrm{f}}=I_{\mathrm{f}, \mathrm{ref}} \frac{\rho}{\rho_{0}}\left(\frac{d}{R}\right)^{2}\left(\frac{N}{N_{\text {ref }}}\right)^{\alpha}
$$

where $I_{\mathrm{f}, \text { ref }}$ is the power flux at a distance $d=0.5 \mathrm{~m}$ from a reference shower of $E_{\text {ref }}=3.36 \times 10^{17} \mathrm{eV}$, $R$ is the distance between the detector and the EAS segment, $\rho\left(\rho_{0}\right)$ is the atmospheric density at the altitude of the EAS segment (at sea level), and $N$ is the number of shower particles in the EAS segment. $N_{\text {ref }}$ is the average number of shower particles at the maximum of the EAS development for a proton primary of energy $E_{\text {ref. }} N$ and $N_{\text {ref }}$ are given by a Gaisser-Hillas [9] parameterization of their respective EAS. The exponent $\alpha$ accounts for the degree of coherence of the MBR emission, ranging from $\alpha=1$ for incoherent to $\alpha=2$ for fully coherent.

For a given pair of $I_{\text {ref }}$ and $\alpha$, events with the same energy and geometry of the selected SD events (Sect. 3.2) were simulated using Eq. 3.1 and a realistic detector response. A pair $\left(I_{\text {ref }}, \alpha\right)$ was excluded at $95 \%$ CL when the corresponding number of simulated events with SLT was less than 3 [10]. Limits on the MBR parameters are shown in Fig. 5, which improve significantly over previous MIDAS results [11] and unequivocally rule out the signal reported in [3] as MBR emission. Recent calculations [12] predict an incoherent MBR emission a factor 200 smaller than in [3], which would require a further improvement in the experimental sensitivity to be detected.

\section{Discussion and conclusions}

Two setups, GIGAS and MIDAS, aiming at the observation of the MBR in coincidence with event detected at the Pierre Auger Observatory were presented. While clear radio events were observed with GIGAS, no signal were found with MIDAS microwave telescope. These results are however compatible if one considers other emission origins. Indeed, the radio signal observed with 

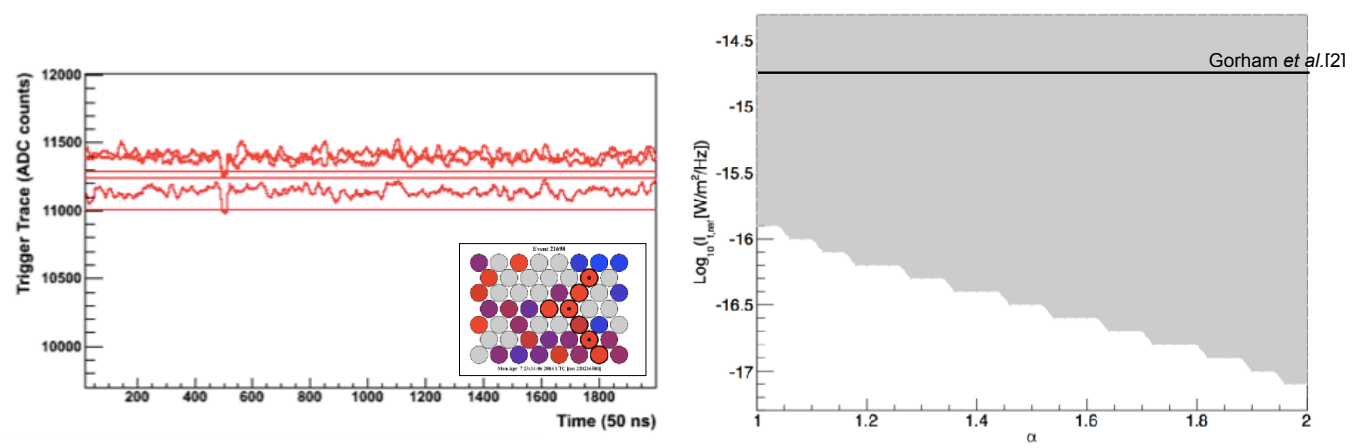

Figure 5: Left: MIDAS candidate event waveforms and pixel configuration. Right: $95 \%$ CL limits in the plane of the reference intensity $\left(I_{\text {ref }}\right)$ and the energy scaling $(\alpha)$ (see Eq. 3.1)

GIGAS are all from close shower axis and might be attributed to coherent emissions known to be dominant at lower frequencies [2]. This would also explain the absence of event observation in the MIDAS data since this detector views the shower from the side and is not optimized for the detection of short radio signals.

These results are in agreement with previous non detection of MBR in beam experiments $[4,5]$ and in situ experiment [6]. They ruled out for the first time all the parameters used for the interpretation in terms of MBR of the original beam test experiment. The intensity of the MBR found by subsequent calculations [12] is smaller by at least a factor 200 and is still uncertain. Going down to this level of sensitivity would require deeper developments such as a cryo-cooled system and is beyond the initial goal of a simple, inexpensive and mass sensitive system.

\section{Acknowledgement}

We acknowledge the support of the French Agence Nationale de la Recherche (ANR) under reference ANR-12- BS05-0005-01.

\section{References}

[1] D. Martello, for the Pierre Auger Observatory, these proceedings.

[2] F. Schröder, Prog. Part. Nucl. Phys. 93 (2017) 1-68.

[3] P. Gorham et al., Phys. Rev. D 78 (2008) 032007.

[4] J. Alvarez-Muñiz et al., RICAP2014, EPJ 121 (2016) 03010.

[5] C. Williams et al., EPJ 53 (2013) 08008.

[6] R. Šmida et al., Phys. Rev. Lett. 113 (2014) 221101.

[7] J. Alvarez-Muñiz et al., Nucl. Instrum. Meth. A 719 (2013) 70-80.

[8] M. Richardson, Vanderbilt University, PhD thesis (2016).

[9] T.K. Gaisser and A.M. Hillas, Proc. 15th Int. Cosmic Ray Conf., Plovdiv, Bulgaria, 8 (1977) 353.

[10] G. Feldman and R. Cousins, Phys. Rev. D 57 (1998) 3873-3889.

[11] J. Alvarez-Muñiz et al., Phys. Rev. D 86 (2012) 051104.

[12] I. Al Samarai et al., Phys. Rev. D 93 (2016) 052004. 